\title{
TU/e Emphoven

\section{Attractor crisis and bursting in a fluid flow with two no-slip directions}

Citation for published version (APA):

Molenaar, D., Clercx, H. J. H., Heijst, van, G. J. F., \& Yin, Z. (2007). Attractor crisis and bursting in a fluid flow with two no-slip directions. Physical Review E - Statistical, Nonlinear, and Soft Matter Physics, 75(3), 0363091/7. [036309]. https://doi.org/10.1103/PhysRevE.75.036309

DOI:

10.1103/PhysRevE.75.036309

Document status and date:

Published: 01/01/2007

\section{Document Version:}

Publisher's PDF, also known as Version of Record (includes final page, issue and volume numbers)

\section{Please check the document version of this publication:}

- A submitted manuscript is the version of the article upon submission and before peer-review. There can be important differences between the submitted version and the official published version of record. People interested in the research are advised to contact the author for the final version of the publication, or visit the $\mathrm{DOI}$ to the publisher's website.

- The final author version and the galley proof are versions of the publication after peer review.

- The final published version features the final layout of the paper including the volume, issue and page numbers.

Link to publication

\section{General rights}

Copyright and moral rights for the publications made accessible in the public portal are retained by the authors and/or other copyright owners and it is a condition of accessing publications that users recognise and abide by the legal requirements associated with these rights.

- Users may download and print one copy of any publication from the public portal for the purpose of private study or research.

- You may not further distribute the material or use it for any profit-making activity or commercial gain

- You may freely distribute the URL identifying the publication in the public portal.

If the publication is distributed under the terms of Article 25fa of the Dutch Copyright Act, indicated by the "Taverne" license above, please follow below link for the End User Agreement:

www.tue.nl/taverne

Take down policy

If you believe that this document breaches copyright please contact us at:

openaccess@tue.nl

providing details and we will investigate your claim. 


\title{
Attractor crisis and bursting in a fluid flow with two no-slip directions
}

\author{
D. Molenaar, ${ }^{*}$ H. J. H. Clercx, and G. J. F. van Heijst \\ Department of Physics, Eindhoven University of Technology, P.O. Box 513, 5600MB Eindhoven, The Netherlands \\ Z. Yin \\ National Microgravity Laboratory, Institute of Mechanics, Chinese Academy of Sciences, 15 Bei Si Huan Xi Lu, \\ Beijing 100080, People's Republic of China
}

(Received 6 February 2006; revised manuscript received 7 November 2006; published 26 March 2007)

\begin{abstract}
Characteristic burtsing behavior is observed in a driven, two-dimensional viscous flow, confined to a square domain and subject to no-slip boundaries. Passing a critical parameter value, an existing chaotic attractor undergoes a crisis, after which the flow initially enters a transient bursting regime. Bursting is caused by ejections from and return to a limited subdomain of the phase space, whereas the precrisis chaotic set forms the asymptotic attractor of the flow. For increasing values of the control parameter the length of the bursting regime increases progressively. Passing another critical parameter value, a second crisis leads to the appearance of a secondary type of bursting, of very large dynamical range. Within the bursting regime the flow then switches in irregular intervals from the primary to the secondary type of bursting. Peak enstrophy levels for both types of bursting are associated to the collapse of a primary vortex into a quadrupolar state.
\end{abstract}

DOI: 10.1103/PhysRevE.75.036309

PACS number(s): 47.15.Rq, 05.45.Ac

\section{INTRODUCTION}

Attractor crises are global bifurcations, resulting in the destruction of an existing chaotic attractor and, possibly, in temporal intermittent switching between different types of chaotic behavior $[1,2]$. These bifurcations occur under small changes of the system parameter, when the evolution is already in a chaotic regime. Hence crisis-induced intermittency is rather different from the classical intermittency described by Manneville and Pomeau [3], where a system switches between a periodic orbit and chaotic bursts. Experimental and numerical observations of crises and crisisinduced intermittency are numerous, ranging from essentially two-dimensional systems like semiconductor lasers [4-6] to higher-dimensional systems like numerical solar dynamo models [7] or experimental (super)fluid convection [8]. In two-dimensional systems crises are the result of a tangency of the stable and unstable manifolds (directions) of saddle points within a chaotic attractor. For systems with more than two dimensions, it was recently argued by Alligood et al. [9] that a tangency is not necessary for a crisis to occur. These authors introduced the crossing bifurcation, in which the unstable manifold of one fixed point intersects the stable manifold of another fixed point, as a possible mechanism for an attractor crisis in high-dimensional systems.

Here we present numerical results indicating an attractor crisis in a high-dimensional fluid dynamics problem, namely a driven, viscous fluid flow in a bounded no-slip domain with two spatial directions. These purely two-dimensional (2D) direct numerical simulations on domains with no-slip walls [10-13] form an intermediate step between the traditional computational setting of 2D flows on a double-periodic domain and laboratory experiments on quasi-2D flows in con-

\footnotetext{
*Present address: Physics of Geological Processes, University of Oslo, P.O. Box 1048, Blindern, 0316 Oslo, Norway.
}

tainers with rigid walls $[14,15]$. For both laboratory experiments and numerical simulations, the transition sequence from steady to chaotic and, eventually, turbulent solutions as a function of an external control parameter is of intrinsic interest. This issue was studied both in quasi-2D laboratory experiments in square [16] and elongated domains [17] and in $2 \mathrm{D}$ computations, for the case of periodic boundary conditions [18] and for stress-free conditions in one direction and periodic conditions in the other direction [19], revealing a rich variety in behavior.

For the case of a driven flow in a square no-slip domain, it was shown by means of direct numerical simulations that the transition from steady to chaotic solutions takes place through the destruction of invariant tori [20]. Using a steady forcing, we consider solutions as a function of the rescaled inverse kinematic viscosity parameter. Starting with the flow on a strange or chaotic attractor, we study this system further in the present work. Gradually increasing the value of the control parameter a global bifurcation occurs, in the form of an attractor crisis. The crisis is caused by a collision of the strange attractor with four saddle points, and results in a complicated dynamics. For parameter values immediately beyond the crisis value, the system displays a dual behavior. Initially, characteristic bursting-type behavior occurs, associated to the interplay between the chaotic attractor and the saddles. From the unstable manifold of a saddle point the trajectory is ejected into the surrounding phase space through a burst event, and then bounces back to re-enter the chaotic domain via another saddle point, in a heteroclinic connection. The bursting regime terminates when the system reaches its asymptotic attractor, the chaotic set associated to the precrisis flow. The length of the transient bursting regime increases exponentially with the value of the control parameter.

However, increasing the control parameter beyond a critical value, a secondary type of bursting appears, of very large dynamical range. Within the transient bursting regime, the flow may then switch from the primary to the secondary type 
of bursting, before the bursting regime is terminated and the flow reaches the chaotic asymptotic attractor. A burst of the second type occurs when the flow enters an unstable manifold of the asymptotic chaotic set, from which it is ejected into the outer parts of phase space.

Both types of bursting are associated to the breakup of a large coherent monopolar vortex into a quadrupolar structure, where the primary vortex exists during the laminar phases preceding a burst. However, the number of smaller, secondary vortices differs for the two types of bursting.

The following section contains an overview of the balance equations and computational methods. In Sec. III we present our observations and Sec. IV contains a brief discussion and our conclusions.

\section{BALANCE EQUATIONS AND COMPUTATIONAL MODEL}

In the two-dimensional domain $\mathcal{D}:=\{x, y \mid-d / 2 \leq x \leq d / 2$, $-d / 2 \leq y \leq d / 2\}$, a fluid flow has Eulerian velocity $\mathbf{u}(t, \mathbf{x})$ $=(u(t, \mathbf{x}), v(t, \mathbf{x}))$, where $t \in \mathbb{R}^{+}$and $\mathbf{x} \in \mathcal{D}$. The fluid has a unit density, $\rho=1$, and the velocity $\mathbf{u}(t, \mathbf{x})$ is area preserving, due to the incompressibility condition

$$
\nabla \cdot \mathbf{u}=0 .
$$

The associated vorticity equation describes a dissipative dynamical system

$$
\partial_{t} \omega+(\mathbf{u} \cdot \nabla) \omega-\nu \Delta \omega=q,
$$

with kinematic viscosity parameter $\nu>0$ and external forcing $q(\mathbf{x})$. The scalar vorticity is defined as $\omega(t, \mathbf{x})=\partial_{x} v(t, \mathbf{x})$ $-\partial_{y} u(t, \mathbf{x})$. In the nondimensional form of Eq. (2), the kinematic viscosity $\nu$ is replaced by the integral-scale Reynolds number, Re: $=\frac{1}{2} U d / \nu$, where $U$ is a spatially averaged (rootmean-square) velocity scale. On the domain boundary $\partial \mathcal{D}$, the natural, no-slip conditions are applied,

$$
\mathbf{u}(t, \mathbf{x})=0 \quad \text { for } \mathbf{x} \in \partial \mathcal{D} .
$$

The flow is brought to life from zero-vorticity initial conditions, $\omega_{0}(\mathbf{x})=0$, by the stationary external forcing, which consists of two parts. At a fixed wave number, $k \in Z^{+}$, Fourier modes are excited in both spatial directions, with a fixed vorticity amplitude, $A_{0} \in \mathbb{R}$. The spatial configuration associated to such a forcing is a chessboard pattern of $k^{2}$ driven vortices of alternating sign. Furthermore, a small solid body rotation of amplitude $A_{s b} \simeq 0.2 A_{0}$ is added. Note that the double-periodic forcing field, mapped onto $\mathcal{D}$, is not required to obey the no-slip conditions.

Fixing, then, the parameters

$$
k=6, \quad A_{0}=0.05, \quad \text { and } d=2,
$$

only the kinematic viscosity parameter $\nu$ is varied, a codimension-1 problem. This latter quantity is normalized with the forcing amplitude, such that the control parameter becomes

$$
\nu^{\prime}=\left(A_{0} / \nu\right) .
$$

We study the behavior of the system in the parameter interval $80.0 \leq \nu^{\prime} \leq 150.0$, analyzing the temporal evolution in terms (a)
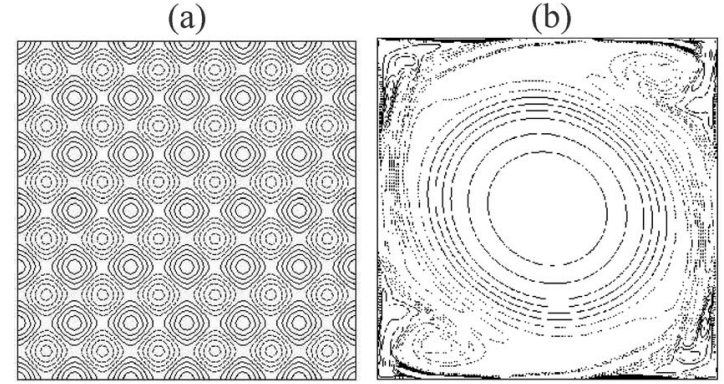

FIG. 1. Snapshots of vorticity isolines for the ground state $\nu^{\prime}$ $=20.0$ (a), resembling the forcing field $q(\mathbf{x})$, and for the precrisis chaotic solution, at parameter value $\nu^{\prime}=80.0$ and time $t=807.5$ (b).

of time series of the (nondimensional) enstrophy, which is defined as

$$
Z(t)=\frac{1}{2} \int_{\mathcal{D}} \omega^{2} d A,
$$

and point-measured time series of the vorticity, $\omega(t, \mathbf{x})$.

A pseudospectral code based on Chebyshev polynomials is used to solve the vorticity equation [21], using an AdamsBashforth Crank-Nicolson time stepping scheme. To speed up computations, a modest parallellization is applied, using a parallel task-distribution scheme, where the computation of the nonlinear term is distributed to four processors for each time step [22]. Our simulations are well resolved and the resolution varies from $N=161$ with $\delta t=3.4 \times 10^{-4}$, at the lower end of the parameter range, to $N=257$ with $\delta t=1.3$ $\times 10^{-4}$, at the upper end of the range. Here, $N$ denotes the number of modes in either direction and $\delta t$ denotes the time step. It must be noted that the structure of the numerical code prevents the propagation of round-off errors, such that an initial excitation of even-even modes can only generate nonzero odd-odd modes and vice versa. A small amount of random noise, which is not added here, should suffice to excite the remaining odd-even and even-odd modes. Integration times run up to $t=1000.0$, yielding record lengths on the order of $10^{5}$ data points.

\section{CRISIS AND BURSTING}

\section{A. Primary bursting}

In a square domain with no-slip boundaries, the transition from a stationary ground state, with constant enstrophy and vorticity values, to time-dependent chaotic motion takes place through the destruction of invariant tori [20]. The transition sequence is similar to the well-known Ruelle-Takens scenario $[23,24]$. From the ground state, which resembles the forcing field $q(\mathbf{x})$, Fig. 1(a), a steady bifurcation leads to a secondary time-independent solution, followed by a Hopf bifurcation to a period-1 limit cycle. Subsequently, a second and a third fundamental frequency enter the flow through respective Hopf bifurcations, after which the resulting quasiperiodic flow breaks down and gives way to chaotic temporal behavior. These transitions are found as the control parameter is increased from $\nu^{\prime}=25.0$, for the secondary steady solutions, to $\nu^{\prime}=80.0$, for the chaotic flow. 


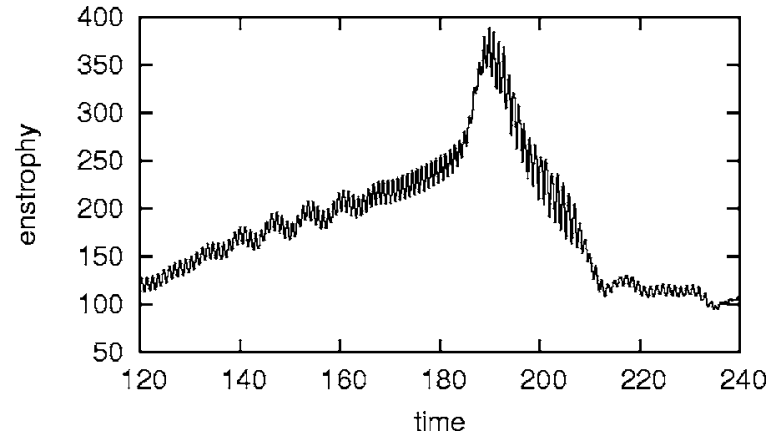

FIG. 2. Enstrophy time series showing the primary bursting behavior appearing after the crisis, for parameter value $\nu^{\prime}=83.75$. Quantities are dimensionless.

The flow field associated to the chaotic motions is spatially coherent and consists of a large, primary vortex in the center of the domain, surrounded by smaller, secondary vortices, Fig. 1(b). The primary vortex is inherited from the preceding (quasi)periodic solutions [20]. As Eqs. (1) and (2), with forcing field $q(\mathbf{x})$ and no-slip boundary conditions (3), are equivariant with respect to reflections and rotations of the square, the symmetry group $\mathbf{D}_{4}$ is associated to the flow field in the ground state. However, the $\mathbf{D}_{4}$ symmetry is broken at the secondary Hopf bifurcation, leaving a symmetry with respect to rotations over an angle $\pi$ about the origin $\mathbf{x}$ $=(0,0)$, Fig. 1(b). The latter symmetry corresponds to the action $R_{\pi}$,

$$
R_{\pi}:(x, y) \rightarrow(-x,-y)
$$

and, together with the identity $R_{\pi}^{2}=I$, forms the symmetry group $\mathbf{Z}_{2}:=\left\{I, R_{\pi}\right\}$.

The secondary vortices occur as a pair, in line with the $R_{\pi}$ symmetry of the flow configuration, see Fig. 1(b) for a snapshot of a typical vorticity field. These vortices result from the rollup of detached boundary layers. Throughout the evolution the flow configuration remains unaltered and the chaotic character of the temporal signal is caused by wobbling motions of the primary vortex structure. Within the chaotic regime, the enstrophy oscillates irregularly around a mean value close to $\langle Z\rangle \simeq 500$.

Increasing the value of the control parameter to $\nu^{\prime}$ $=83.75$, the time series initially display a very different, short-lived bursting-type behavior, referred to as the primary bursting. The transient bursting regime terminates when the flow returns to the usual chaotic regime, in which it remains during the further temporal evolution of the system. An example of a primary burst is shown in Fig. 2, as recorded in the enstrophy time series. One clearly observes oscillating motions of limited amplitude, a so-called laminar phase, before the enstrophy of the flow increases sharply in a burst event. Subsequently, the enstrophy bounces back to its preburst value, to enter another laminar phase.

During the laminar phase the flow configuration consists of a large, primary vortex structure in the center of the domain, Fig. 3(a). The number of secondary vortices of opposite sign has increased with respect to the flow on the strange attractor, from one to three distinct pairs. E.g., compare with (a)

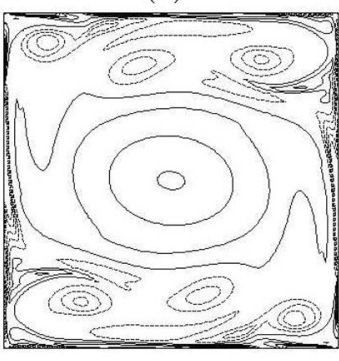

(c)

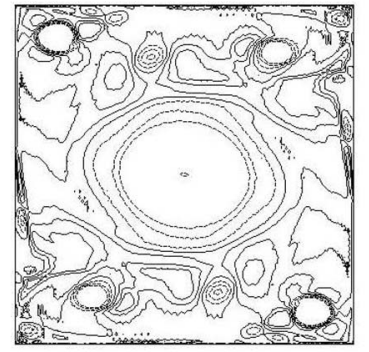

(b)

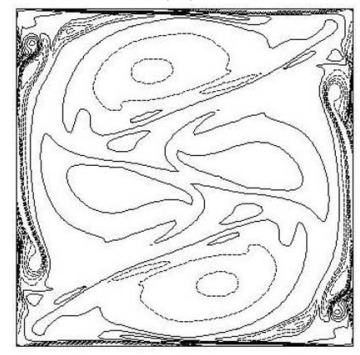

(d)

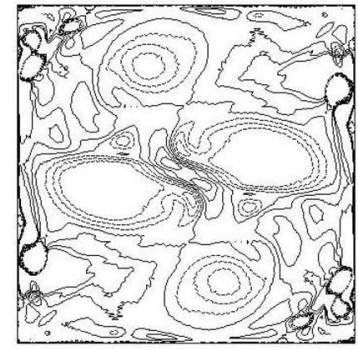

FIG. 3. Isovorticity contour plots for parameter value $\nu^{\prime}$ $=83.75$, the corresponding enstrophy time series is shown in Fig. 2 . These snapshots display the flow configuration for a primary laminar phase at $t=142.8$ (a) and burst event at $t=190.4$ (b). Contour lines range from -30 to 30 with an interval of 6 ; negative values are represented by dotted lines. For both vorticity contour plots the associated Weiss function is shown in (c), for $t=142.8$, and in (d), for $t=190.4$. Clearly, during a burst event the character of the flow in the center of the domain changes from elliptic (solid lines) to hyperbolic (dotted lines). Contour lines in (c) and (d) range from -90 to 90 with an interval of 30 ; negative values are represented by dotted lines.

the contour plot of the vorticity field in the chaotic regime in Fig. 1(b). These satellite vortices are advected along with the central structure.

Bursting behavior, attributed to large-scale selforganization, is a well documented phenomenon for twodimensional bounded flows with many degrees of freedom [25]. A recent overview of several types of bursting behavior in fluid dynamics problems is given in Ref. [26]. In the present case, peak enstrophy levels, attained, for instance, at $t=190.4$ in Fig. 2, coincide with a remarkable, temporary collapse of the primary vortex structure into a quadrupolar configuration, visualized in a vorticity contour plot in Fig. 3(b). The temporary collapse of the primary vortex leads to a change in the nature of the flow in the center of the domain, as shown by means of the Weiss function [27] in Figs. 3(c) and 3(d). Defined as

$$
Q(t, \mathbf{x})=-4 \operatorname{det} \nabla \mathbf{u},
$$

the value of the latter quantity is negative for elliptic areas, corresponding to vortex cores, and positive for hyperbolic areas, corresponding to the surrounding, irregular flow field. From the figure it follows that during a burst event the flow in the center of the domain changes in character, from elliptic, Fig. 3(c), to hyperbolic, Fig. 3(d). 


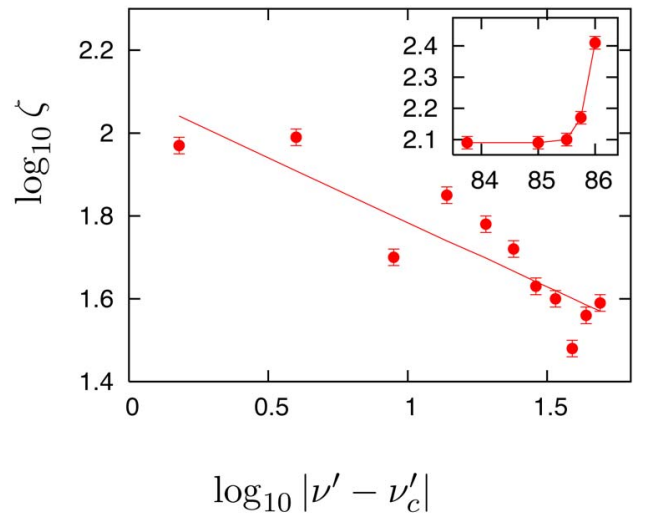

FIG. 4. (Color online) Log-log plot of the average duration $\zeta$ of the laminar phases against $\left|\nu^{\prime}-\nu_{c}^{\prime}\right|$ (dots). The solid line is a leastsquares fit to the data, with slope $\gamma=-0.31 \pm 0.06$. A semilogarithmic plot of the duration of the primary bursting regime $\tau$, showing $\log _{10}(\tau)$ as a function of the value of the control parameter $\nu^{\prime}$ (inset).

The duration of the transient bursting regime $\tau$ is a monotically increasing function of the control parameter. An exponential increase in the value of $\tau$ is clearly observed in the inset in Fig. 4, which plots $\log _{10}(\tau)$ as a function of the control parameter. Thus numerical integration times must increase accordingly, if the chaotic regime, which is the asymptotic attractor of the flow and which appears once the transient regime terminates, is to be observed for large parameter values.

Furthermore, for increasing values of the control parameter the bursting events of the primary type increase in frequency and the laminar periods become progressively shorter. Eventually, for parameter values beyond the range discussed here, the bursting behavior disappears from the dynamics, e.g., for $\nu^{\prime}>150$ the flow lies entirely on a large chaotic attractor. A general aspect of many types of bursting behavior is the existence of a power-law scaling of the average time between the bursts with the control parameter [26]. Denoting the duration of the laminar phase as $\zeta$, the scaling takes the form of a power law,

$$
\zeta \propto\left|\nu^{\prime}-\nu_{c}^{\prime}\right|^{\gamma},
$$

where $\nu_{c}^{\prime}$ is the critical parameter value for which bursting behavior first occurs.

A problem for our computations is that the (critical) value $\nu_{c}^{\prime}$ could not be determined exactly, an issue which remains to be addressed. In Fig. 4, where we used the estimate $\nu_{c}^{\prime}$ $=83.5$, power-law scaling behavior is clearly observed, with estimated scaling exponent $\gamma=-0.31 \pm 0.06$. Changing the estimate for the value of $\nu_{c}^{\prime}$ to, for instance, the lower bound $\nu_{c}^{\prime}=83.0$ does not lead to large differences in the scaling exponent, yielding $\gamma \simeq-0.35$.

Global bifurcations leading to sudden discontinuities in the behavior of a chaotic attractor [1,2], as observed in our computational results, are known as attractor crises. Hence our observations so far suggest a scenario where an attractor crisis occurs within the parameter interval $82.5<\nu^{\prime} \leq 83.75$, due, as is shown in Sec. III C, to the collision of an existing

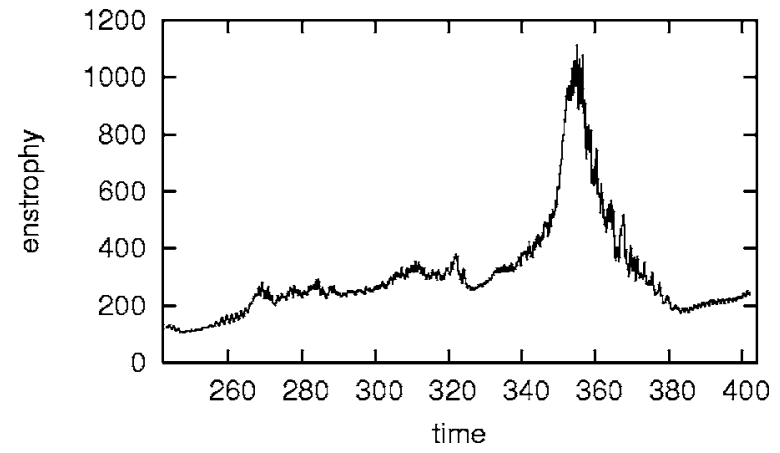

FIG. 5. Enstrophy time series, displaying a secondary laminar phase and bursting, for parameter value $\nu^{\prime}=87.0$. Note the large dynamical range of the secondary bursting, for which peak levels are attained around $t=355.0$, as compared to the primary type bursting shown in Fig. 2. Quantities are dimensionless.

strange attractor with four saddles. Beyond the critical parameter value at which the crisis occurs, the system displays the transient bursting behavior, associated to the saddles. The ghost of the precrisis attractor then forms the asymptotic attractor of the flow, as it returns to the usual chaotic motions once the transient regime terminates. In our computations we did not detect a subsequent return to the bursting regime.

\section{B. Secondary bursting}

For values of the control parameter up to $\nu^{\prime}=86.0$ the flow evolves, as discussed above, from a transient bursting regime onto a set of chaotic motions. However, in the parameter interval $86.0<\nu^{\prime} \leq 86.15$ another, markedly different type of bursting behavior appears within the transient regime, indicating a second crisis has occurred. For this secondary type of bursting the laminar phases are similar in character to the precrisis chaotic flow, followed by a burst event of very large dynamical range, of up to five times the mean precrisis enstrophy value. A progression from such a laminar phase to a bursting event is shown in an enstrophy time series, Fig. 5.

Thus the transient bursting regime now contains two types of bursting behavior, between which the flow switches in irregular intervals. Eventually, the flow may still leave the bursting regime altogether and end up on a set of chaotic motions, as is observed for several values of the control parameter. Consequently, the scaling of the length of the bursting regime, shown in the inset of Fig. 4 for the primary bursting regime, is no longer valid when the secondary bursting appears. Furthermore, for the secondary type of bursting no clear scaling of the length of the laminar phases with the value of the control parameter was observed, in contrast to the primary type of bursting.

The physical manifestation of the secondary bursting lies in the formation and temporary destruction of a large coherent vortex structure, filling the center of the domain. During the laminar phase, Fig. 6(a), wobbling motions of the primary vortex cause an irregular temporal signal, similar to the flow configuration on the precrisis chaotic attractor. At the onset of a burst, secondary vortices, formed in opposite cor- 
(a)

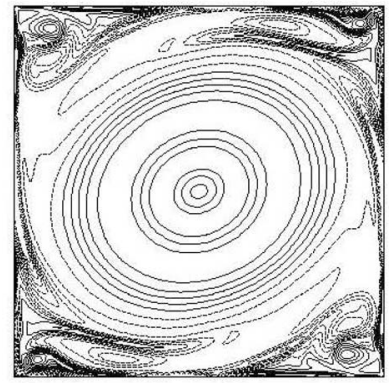

(b)

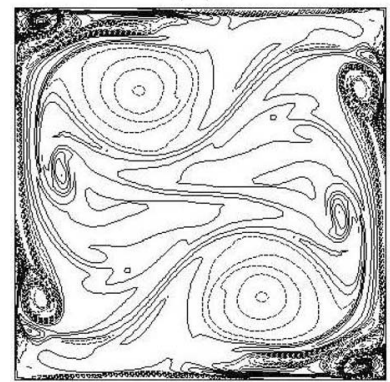

(c)

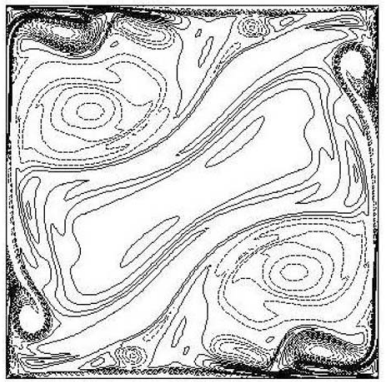

FIG. 6. Isovorticity contour plots, for parameter value $\nu^{\prime}=87.0$, corresponding to the vorticity time series in Fig. 5. Three consecutive plots show the flow field during the laminar phase at $t=326.4$ (a), close to enstrophy peak levels at $t=353.6$ (b) and during the return to a laminar phase at $t=360.4$ (c). Eleven equidistant contour lines are shown in each case, ranging, respectively, from -30 to 30 , from -50 to 50 and from -30 to 30 ; negative values are represented by dotted lines.

ners of the domain, gain sufficient strength to squeeze the primary structure into a tripolar and, finally, a quadrupolar configuration, Fig. 6(b). The latter configuration coincides with peak enstrophy levels, observed, for instance, at $t$ $=355.0$ in Fig. 5. Upon the following return from peak levels, the flow field re-enters the tripolar configuration, shown in Fig. 6(c), and, finally, its primary vortex state.

\section{Phase-space reconstruction}

A phase-space reconstruction of the precrisis chaotic flow is shown in Fig. 7(a). Here, the phase plot is projected on the $(\omega(t, \mathbf{x}), \omega(t+\tau, \mathbf{x}))$ plane, where the delay $\tau$ is determined from the mutual average information [28]. We used pointmeasured vorticity time series $\omega(t, \mathbf{x})$, with $\mathbf{x}=(0,1 / 2)$, to reconstruct the phase space, noting that time series measured at different locations do not contain additional information. The associated Poincaré return map is displayed in Fig. 7(b), and shows the (first) return coordinates of the phase-space trajectory to a two-dimensional slice through threedimensional phase-space. The chaotic flow results from the destruction of invariant tori [20] and the associated return map possesses no clear structure. Note that while the flow field is $R_{\pi}$ symmetric, the attractor does not have such symmetry, Fig. 7(a).

Clearly, our dynamical system of bounded and forced 2D flows experiences a sharp increase in attractor size at the
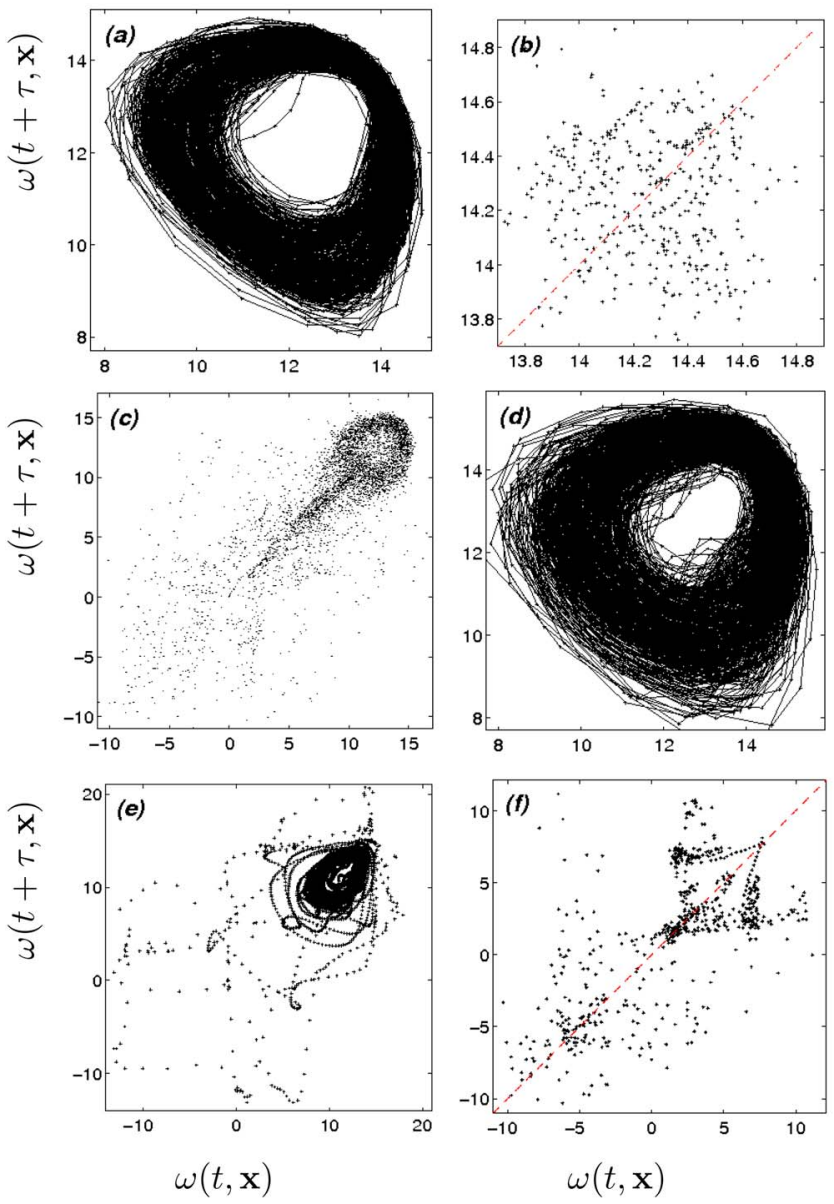

FIG. 7. (Color online) Projection of the phase portrait of the precrisis chaotic attractor, at parameter value $\nu^{\prime}=80.0$ (a), onto the $(\omega(t, \mathbf{x}), \omega(t+\tau, \mathbf{x}))$ plane, where $\mathbf{x}=(0,1 / 2)$. A Poincaré first return map is shown in (b), where the bisecting line serves to guide the eye. A postcrisis phase portrait, for $\nu^{\prime}=85.0$, is shown in (c) and a blowup of the portrait, corresponding to the area of the asymptotic attractor is shown in (d). Phase portrait of a secondary burst (e), for parameter value $\nu^{\prime}=86.5$. A Poincaré first return map for $\nu^{\prime}=90.0$ is displayed in (f), the bisecting line serves to guide the eye. For visualization purposes the number of data points is reduced in all shown phase portraits.

crisis. E.g., compare a post-crisis phase portrait, for $\nu^{\prime}$ $=85.0$, Fig. $7(\mathrm{c})$, where the axis are rescaled to plot the at tractor, to the precrisis phase portrait, Fig. 7(a). The dense region on the attractor corresponds to the chaotic regime, which sets in upon termination of the transient bursting regime and forms the asymptotic attractor of the flow. A magnification of the portrait, found at $\nu^{\prime}=85.0$, displays the dense set corresponding to the asymptotic attractor, Fig. 7(d). This set is, roughly, contained in the interval 9 $<\omega(t, \mathbf{x}), \omega(t+\tau, \mathbf{x})<15$ in the projection onto $\mathbb{R}^{2}$. Note also that the set is more densely filled as compared to the precrisis attractor, Fig. 7(a), which corresponds to a more irregular behavior in the temporal signal.

The initial crisis is caused by the collision of the precrisis chaotic attractor with four saddles. In the portrait, Fig. 7(c), the saddles, which give rise to the initial bursting regime, are located within the nondense set centered around, roughly, 
$(2.5,2.5)$. The resolution in the portrait is decreased for visualization purposes.

The phase-space trajectory winds in an anticlockwise fashion around the four saddle points. When the initial bursting regime terminates, the flow moves towards a set of chaotic motions, which form the asymptotic attractor of the system, through a heteroclinic connection via one of the saddle points. A phase portrait of a secondary burst is shown in Fig. 7(e). Here, the laminar motions correspond to the dense phase-space area of the precrisis attractor, from where the trajectory is ejected in a burst event. Hence the system enters an unstable manifold of the precrisis chaotic set, spends a short time interval on this unstable manifold, and is subsequently ejected into out-lying parts of phase space. This picture is confirmed by the flow configuration during the laminar state, which resembles the precrisis configuration, Fig. 6(a).

At parameter value $\nu^{\prime}=90.0$ an extended primary bursting regime occurs. For this system the Poincaré first return map is shown in Fig. 7(f). The laminar phases lead to some structure in the return map, although there are no clear tangencies. An important observation is that, in general, the Poincaré ( $n$ th) return maps and the angular return maps of the postcrisis system (not shown) do not reveal any clear structure indicating tangencies.

The correlation dimension, estimated according to Ref. [29], is close to $d_{\text {corr }} \simeq 4$ for most values of the control parameter. Furthermore, estimates for the embedding dimension $d_{e m b}$, the smallest integer dimension needed to fully unfold the dynamics [2], suggest that the attractor associated to the bursting motions lies in $\mathbb{R}^{4}$.

\section{DISCUSSION AND CONCLUSIONS}

A crisis may result in intermittent dynamics, where the post-crisis system either switches in semiregular intervals between the ghost of the precrisis attractor and the phase-space area which became available to the system during the crisis, or passes a transient regime before entering the precrisis phase-space area. In two dimensions crises are associated to heteroclinic or homoclinic tangencies [2] and are associated with well-defined scaling of, for instance, the average time between bursts.

For systems with phase-space dimension 3, a tangency may not be necessary for a crisis to occur [9], which forms a possible explanation for the lack of tangencies in our observations. A key point is that higher-dimensional systems may display unstable dimensional variability (UDV), for which different saddle points have a different number of unstable directions. To directly establish UDV in our system longer integration times are needed, an issue we hope to address in future work. Note that the dynamics described in the previous section is markedly different from the so-called multichannel intermittency. The latter intermittency corresponds to a classical type-I intermittency with two different laminar phases (reinjection channels) induced by the presence of phase-space symmetry $[30,31]$. However, in the present case the intermittency sets in after an attractor crisis and, as argued above, neither the ( $n$ th) Poincaré return maps nor the angular return maps reveal the tangencies associated to multichannel intermittency.

Another point is the possibility of intermittency caused by a chaos-hyperchaos transition, where hyperchaos refers to an attractor with two positive Lyapunov exponents [32]. Such a transition should be detectable in the system Poincaré map, which is dense in the chaotic regime and has both dense and nondense regions in the hyperchaotic regime (as in the present case). In addition, the correlation integral shows a progression from one to two scaling regimes as a function of separation length for the chaos-hyperchaos transition. However, the latter property was not observed for our system, effectively ruling out the chaos-hyperchaos bifurcation as the mechanism causing bursting.

A difficulty for the presented observations is that the respective parameter values for which the primary and the secondary type of bursting first appear cannot be determined exactly. Furthermore, longer integration times may be needed to establish firmly whether scaling of the duration of secondary laminar phases is lacking or not.

Summarizing, our direct numerical simulations of a confined 2D fluid flow, reveal that an attractor crisis occurs, in which a chaotic attractor collides with a set containing four saddle points. Following the crisis, located in the parameter interval $82.5<\nu^{\prime} \leq 83.75$, the flow initially moves along the saddles. Primary bursting then takes place when the flow is ejected from the set, via the unstable manifold of one of the saddle points, and subsequently re-enters through a different saddle point. The average time between the primary bursts decreases as the value of control parameter increases, with scaling exponent $\gamma=-0.31 \pm 0.06$. At each parameter value, the bursting regime is terminated when the flow reaches the asymptotic attractor, the ghost of the precrisis chaotic set.

However, in the parameter interval $86.0<\nu^{\prime} \leq 86.15$ a secondary type of bursting appears, signalling an additional crisis and enlargement of the attractor. The secondary bursting is of large dynamical range, and occurs when the flow enters an unstable manifold of the precrisis chaotic set, from which it is ejected into out-lying parts of phase space.

The physical manifestation of both types of bursting is associated to the evolution of a strong primary monopolar vortex structure, which forms during the laminar phase preceding a burst. At the onset of the burst smaller, secondary vortices surrounding the primary structure gain in strength. The flow field evolves from a monopolar to a tripolar and, finally, a quadrupolar configuration, when the primary vortex becomes squeezed between the secondary vortices. Peak enstrophy levels are achieved in the quadrupolar state. Hence within the bursting regimes, a repeating sequence occurs in the flow field: monopole $\rightarrow$ tripole $\rightarrow$ quadrupole $\rightarrow$ tripole $\rightarrow$ monopole. Eventually, the system may leave the bursting regime and enter a phase of chaotic, nonbursting motions, for which the flow field once more consists of a large monopolar vortex.

\section{ACKNOWLEDGMENTS}

One of us (D.M.) would like to thank the Foundation for Fundamental Research on Matter (FOM) for financial sup- 
port. This work was sponsored by the Stichting Nationale Computerfaciliteiten (National Computing Facilities Foundation, NCF) for the use of supercomputer facilities, with fi- nancial support from the Nederlandse Organisatie voor Wetenschappelijk Onderzoek (Netherlands Organization for Scientific Research, NWO).
[1] C. Grebogi, E. Ott, F. Romeiras, and J. A. Yorke, Phys. Rev. A 36, 5365 (1987).

[2] E. Ott, Chaos in Dynamical Systems (Cambridge University Press, Cambridge, England, 2002).

[3] P. Manneville and Y. Pomeau, Physica D 1, 219 (1980).

[4] E. F. Manffra, I. L. Caldas, R. L. Viana, and H. J. Kalinowski, Nonlinear Dyn. 27, 185 (1997).

[5] S. Wieckzorek, B. Krauskopf, and D. Lenstra, Opt. Lett. 26, 816 (2001)

[6] R. Meucci, E. Allaria, F. Salvadori, and F. T. Arecchi, Phys. Rev. Lett. 95, 184101 (2005).

[7] E. Covas and R. Tavakol, Phys. Rev. E 55, 6641 (1997).

[8] G. Metcalfe and R. P. Behringer, Phys. Rev. A 46, R711 (1992).

[9] K. T. Alligood, E. Sander, and J. A. Yorke, Phys. Rev. Lett. 96, 244103 (2006).

[10] S. Li, D. Montgomery, and W. B. Jones, J. Plasma Phys. 56, 615 (1996).

[11] H. J. H. Clercx, S. R. Maassen, and G. J. F. van Heijst, Phys. Rev. Lett. 80, 5129 (1998).

[12] H. J. H. Clercx, S. R. Maassen, and G. J. F. van Heijst, Phys. Fluids 11, 611 (1999).

[13] S. R. Maassen, H. J. H. Clercx, and G. J. F. van Heijst, Europhys. Lett. 46, 339 (1999).

[14] J. Paret, M. C. Jullien, and P. Tabeling, Phys. Rev. Lett. 83, 3418 (1999).

[15] S. R. Maassen, H. J. H. Clercx, and G. J. F. van Heijst, Phys. Fluids 14, 2150 (2002).

[16] J. Sommeria, J. Fluid Mech. 170, 139 (1986).

[17] P. Tabeling, O. Cardoso, and B. Perrin, J. Fluid Mech. 213,
511 (1990).

[18] F. Feudel and N. Seehafer, Phys. Rev. E 52, 3506 (1995).

[19] R. Braun, F. Feudel, and P. Guzdar, Phys. Rev. E 58, 1927 (1998).

[20] D. Molenaar, H. J. H. Clercx, and G. J. F. van Heijst, Phys. Rev. Lett. 95, 104503 (2005).

[21] H. J. H. Clercx, J. Comput. Phys. 137, 186 (1997).

[22] Z. Yin, H. J. H. Clercx, and D. Montgomery, Comput. Fluids 33, 509 (2004).

[23] D. Ruelle and F. Takens, Commun. Math. Phys. 20, 167 (1971).

[24] S. Newhouse, D. Ruelle, and F. Takens, Commun. Math. Phys. 64, 35 (1978).

[25] D. Molenaar, H. J. H. Clercx, and G. J. F. van Heijst, Physica D 196, 329 (2004).

[26] E. Knobloch and J. Moehlis, in Nonlinear Instability, Chaos, and Turbulence, edited by D. Riahi and L. Debnath (Computational Mechanics Publications, Southampton, 2000) pp. 237287.

[27] J. Weiss, Physica D 48, 273 (1991).

[28] H. D. I. Abarbanel, Analysis of Observed Chaotic Data (Springer-Verlag, Del Mar, 1996).

[29] M. T. Rosenstein, J. J. Collins, and C. J. D. Luca, Physica D 65, 117 (1993).

[30] C. Letellier, P. Werny, J.-M. Malasoma, and R. Gilmore, Phys. Rev. E 66, 036220 (2002).

[31] D. Amroun, M. Brunel, C. Letellier, H. Leblond, and F. Sanchez, Physica D 203, 185 (2005).

[32] T. Kaptaniak, K.-E. Thylwe, I. Cohen, and J. Wojewoda, Chaos, Solitons Fractals 5, 2003 (1995). 